\title{
The Antiproliferative and Colony-suppressive Activities of STAT3 Inhibitors in Human Cancer Cells Is Compromised Under Hypoxic Conditions
}

\author{
JILAI TIAN ${ }^{1,2,3}$, HUI XIAO ${ }^{4}$, RUOHAN WU ${ }^{4}$, YANG CAO ${ }^{5}$, CHENGLONG LI ${ }^{6}$, RONALD XU $^{7,8}$, \\ CHRISTOPHER R. PIERSON ${ }^{9}$, JONATHAN L. FINLAY ${ }^{4,10}$, FANG YANG $^{1,2}$, NING GU $^{1,2}$ and JIAYUH LIN ${ }^{3}$ \\ ${ }^{1}$ State Key Laboratory of Bioelectronics, Jiangsu Key Laboratory for Biomaterials and Devices, \\ School of Biological Science and Medical Engineering, Southeast University, Nanjing, Jiangsu, P.R. China; \\ ${ }^{2}$ Collaborative Innovation Center of Suzhou Nano-Science and Technology, \\ Suzhou Key Laboratory of Biomaterials and Technologies, Suzhou, Jiangsu, P.R. China; \\ ${ }^{3}$ Department of Biochemistry and Molecular Biology, School of Medicine, \\ University of Maryland, Baltimore, MD, U.S.A.; \\ ${ }^{4}$ Center for Childhood Cancer and Blood Diseases, The Research Institute at Nationwide Children's Hospital, \\ Department of Pediatrics, College of Medicine, The Ohio State University, Columbus, OH, U.S.A.; \\ ${ }^{5}$ Department of Hematology \& Cancer Biology Research Center, Tongji Hospital, Tongji Medical College, \\ Huazhong University of Science and Technology, Wuhan, Hubei, P.R. China; \\ ${ }^{6}$ Division of Medicinal Chemistry and Pharmacognosy, College of Pharmacy, \\ The Ohio State University, Columbus, OH, U.S.A.; \\ ${ }^{7}$ Department of Precision Machinery and Precision Instrumentation, \\ University of Science and Technology of China, Hefei, Anhui, P.R. China; \\ ${ }^{8}$ Department of Biomedical Engineering, Comprehensive Cancer Center, \\ The Ohio State University, Columbus, OH, U.S.A.; \\ ${ }^{9}$ Department of Pathology and Laboratory Medicine, Nationwide Children's Hospital, and \\ Departments of Pathology and Biomedical Education \& Anatomy, College of Medicine, \\ The Ohio State University, Columbus, OH, U.S.A.; \\ ${ }^{10}$ Hematology and Oncology, The Research Institute at Nationwide Children's Hospital, \\ Department of Pediatrics, College of Medicine, The Ohio State University, Columbus, OH, U.S.A.
}

\begin{abstract}
Constitutive activation of signal transducer and activator of transcription 3 (STAT3) has been indicated as a novel cancer drug target, since it plays an important role in diverse oncogenic processes including survival, cell proliferation and migration. Emerging STAT3 inhibitors have demonstrated efficacy in cancer cells and animal tumor models. It is well known that most solid tumors are characterized by hypoxia, but it is not clear if hypoxic conditions affect activity of STAT3 inhibitors. To examine this, two STAT3 inhibitors were tested to investigate their
\end{abstract}

Correspondence to: J. Lin, $108 \mathrm{~N}$ Greene Street, Department of Biochemistry and Molecular Biology, School of Medicine, University of Maryland, Baltimore, MD 21201, U.S.A. Tel: +1 4107067469, Fax: +1 4107068297, e-mail: JLin@som.umaryland.edu

Key Words: STAT3 inhibitor, STAT3, hypoxia, drug resistance. inhibitory efficacy in cancer cells grown under hypoxic conditions compared with those without hypoxia. Cell proliferation, colony formation and western blot assays were performed to examine the differences in the cell viability, proliferation and proteins in the STAT3 pathway. Under hypoxic conditions, the half-maximal inhibitory concentration values for both STAT3 inhibitors were increased compared to normoxic conditions in human pancreatic cancer, medulloblastoma and sarcoma cell lines. In addition, the ability of both STAT3 inhibitors to inhibit colony formation in pancreatic cancer, medulloblastoma and sarcoma cell lines was reduced under hypoxic conditions when compared to cells under normoxic conditions. Furthermore, there was an increase in phosphorylated STAT3 levels in cancer cells under hypoxic conditions, suggesting this may be one of the mechanisms of resistance. In summary, the results presented here provide a novel finding of STAT3 inhibitor activity under hypoxic conditions 
and indicate that under such low oxygen conditions, the anticancer efficacy of STAT3 inhibitors was indeed hampered. These results highlight the need to develop new therapeutic strategies to overcome the resistance of cancer cells to STAT3 inhibitors under hypoxic conditions.

The signal transducers and activators of transcription (STATs) are a family of cell signaling proteins that play critical roles in inflammation, proliferation and differentiation $(1,2)$. The STAT family of transcription factors activate a variety of genes such as myelocytomatosis (MYC), survivin, cyclo-oxygenase 2 (COX2) and cyclin D1 (3-5). Activation of STAT3 and its target genes can induce cell-cycle progression, immune evasion, angiogenesis, antiapoptotic effects, tumor invasion and metastasis $(6,7)$. Moreover, constitutive activation of the STAT3 pathway occurs in a variety of cancer types and is typically associated with a poor prognosis $(8,9)$.

Activation of receptor tyrosine kinases (RTKs) or Janus kinases (JAKs) can lead to phosphorylation of specific tyrosine residues on STAT3 (P-STAT3), which then homodimerize through Src homology 2 (SH2) domains and subsequently translocate into the nucleus, where binding to promoter elements of target genes acts to regulate their transcription. STAT3 is seen as an attractive therapeutic target because of its important role in cancer progression and tumorigenesis (10); accordingly, several STAT3 inhibitors have been developed, which can be classified as peptides or peptide-mimetics, non-peptide small molecules, natural products, tyrosine kinase inhibitors or DNA-binding modifiers (10). Our work and that of others has demonstrated that several small molecule STAT3 inhibitors have promising antitumor activity both in vitro and in mouse xenograft models. For example, LLL12, a novel small molecule, can persistently inhibit activated STAT3 and cause apoptosis in a variety of human cancer cells (11), the mechanism for which might involve LLL12 binding directly to the phosphorylated tyrosine 705 binding site of the STAT3 monomer but not to JAK1, JAK2 and TYK2, blocking its recruitment to the receptor and thereby preventing phosphorylation by tyrosine kinases and by interference with dimerization (12).

A novel designed compound, LY5 [5,8-dioxo-6-(pyridin3-ylamino)-5,8- dihydronaphthalene-1-sulfonamide], was confirmed to bind to the STAT3 SH2 domain, downregulating the expression of downstream STAT3 targets, including cyclin D1, B-cell lymphoma $(B C L)$-XL, survivin, and micro-RNA $21(13,14)$.

Of note, hypoxia/anoxia is a well-characterized component of the solid tumor microenvironment. For example, pancreatic cancer develops in microenvironments with high levels of hypoxia (15). Owing to the rapid proliferation of cancer cells, the tumor quickly exhausts nutrients and oxygen supply from the normal vasculature, leading to a hypoxic microenvironment. Tumor tissue hypoxia is well known to be associated with chemoresistance, increased genomic instability, suppression of apoptosis, support of autophagy, enhanced receptor tyrosine kinase-mediated signaling, tumor angiogenesis, vasculogenesis, and the propensity for invasion and metastasis $(16,17)$. Hypoxia can regulate tumor drug sensitivity, especially resistance to commonly used chemotherapeutic drugs, such as cisplatin, carboplatin and doxorubicin, in colorectal, lung, prostate and breast tumors (18). Research has demonstrated that the mechanism for this occurs via regulation of the ATP-binding cassette, sub-family $\mathrm{G}$, member 2 gene $(A B C G 2)$ through the activation of extracellular-regulated protein kinases (ERK) $1 / 2$, hypoxiainducible factor (HIF) $1 \alpha$, and the reactive oxygen species (ROS)-nuclear factor erythroid 2-related factor 2 (NRF2)glutamate-cysteine ligase catalytic subunit (GCLC)glutathione $(\mathrm{GSH})$ pathway $(18,19)$, or others. Different signaling factors may be activated under these hypoxic conditions when compared with the normoxic conditions commonly used in experimental settings.

Although there have been multiple studies on the mechanism of chemoresistance in hypoxic cancer cells, the effects of STAT3 inhibitors under hypoxic conditions have not been studied. Herein, we investigated the effects of the STAT3 inhibitors LLL12 and LY5 on human cancer cell viability and colony formation under markedly hypoxic ( $1 \%$ oxygen) conditions. To our knowledge, this study represents the first report of the impaired ability of STAT3 inhibitors on cell viability and colony formation in pancreatic cancer, medulloblastoma, and sarcoma cell lines under hypoxic conditions relative to cancer cells grown under normoxic conditions.

\section{Materials and Methods}

Reagents, antibodies and equipment. Small-molecular STAT3 inhibitors, LLL12 and LY5, were synthesized at the College of Pharmacy, The Ohio State University (Columbus, OH, USA). The compound as powder was dissolved in sterile dimethyl sulfoxide (DMSO) to make a $20 \mathrm{mmol} / \mathrm{l}$ stock solution. Aliquots of the stock solution were stored at $-20^{\circ} \mathrm{C}$. Primary antibodies were purchased from Cell Signaling Tech (Danvers, MA, USA). Horseradish peroxidase (HRP)-conjugated secondary antibodies were obtained from Santa Cruz Biotechnology (Santa Cruz, CA, USA). Hypoxic chambers (Inviv $\mathrm{O}_{2} 300$ ) were obtained from Baker Ruskinn (The Baker Company, Sanford, ME, USA), and were set at $1 \%$ of $\mathrm{O}_{2}, 5 \%$ of $\mathrm{CO}_{2}$ and $94 \%$ of $\mathrm{N}_{2}$.

Cell lines and culture. Human pancreatic cancer cell lines (Capan1, HPAF-II, HPAC, and MIA PaCa-2) and the human osteosarcoma cell line SJSA-1 were purchased from the American Type Culture Collection (Manassas, VA, USA). Human rhabdomyosarcoma cell line RH30 and the medulloblastoma cell lines UW288-1 and UW426 
Table I. Inhibition of cell viability in human cancer cells treated with STAT3 inhibitor LLL12 or LY5 under normoxic and hypoxic conditions. Human cancer cell lines were treated for 48 hours and the half maximal inhibitory concentration $\left(I_{50}\right)$ values were determined for each inhibitor as described in Materials and Methods.

\begin{tabular}{|c|c|c|c|c|c|c|c|c|c|}
\hline \multirow[t]{2}{*}{ Inhibitors } & \multirow[t]{2}{*}{$\mathrm{O}_{2}$} & \multicolumn{8}{|c|}{$\begin{array}{c}\mathrm{IC}_{50}(\mu \mathrm{M}) \\
\text { Cell line }\end{array}$} \\
\hline & & Capan-1 & HPAF-II & HPAC & MIA PaCa-2 & UW288-1 & UW426 & SJSA-1 & RH30 \\
\hline \multirow[t]{2}{*}{ LLL12 } & $21 \%$ & 0.383 & 0.807 & 0.555 & 2.805 & 0.041 & 0.310 & - & - \\
\hline & $1 \%$ & 1.296 & 1.890 & 2.624 & 6.064 & 0.695 & 1.234 & - & - \\
\hline \multirow[t]{2}{*}{ LY5 } & $21 \%$ & 1.912 & 0.536 & 1.238 & - & 1.375 & 2.003 & 0.907 & 0.606 \\
\hline & $1 \%$ & 2.991 & 2.076 & 3.418 & - & 2.469 & 6.182 & 3.427 & 1.550 \\
\hline
\end{tabular}

were provided by Dr. Peter Houghton and Dr. Corey Raffel (The Research Institute at Nationwide Children's Hospital, Columbus, OH, USA). Capan-1 was maintained in Iscove's modified Dulbecco's medium supplemented with $20 \%$ fetal bovine serum (FBS) and $1 \%$ penicillin/streptomycin (PS) (Gibco, Grand Island, NY, USA), while HPAF-II was supplemented with Eagle's minimum essential medium with $10 \%$ FBS and $1 \%$ PS. The SJSA-1 cell line was cultured in RPMI-1640 medium with 10\% FBS and 1\% PS added. All other cell lines were cultured in Dulbecco's modified Eagle's medium supplemented with $10 \%$ FBS and $1 \%$ PS. All cell lines were cultured in a humidified $37^{\circ} \mathrm{C}$ incubator with $5 \% \mathrm{CO}_{2}$. Cells were cultured in chambers both under normoxic condition (where the $\mathrm{O}_{2}$ level was $21 \%)$ and under hypoxic conditions $\left(\mathrm{O}_{2}\right.$ level kept at $\left.1.0 \%\right)$. All media were all purchased from Gibco.

Cell viability and half maximal inhibitory concentration $\left(I C_{50}\right)$ determinations. Cancer cells were seeded in 96-well plates in triplicate at a density of 3,000 cells per well, and kept overnight to adhere. The cells were then treated with five different concentrations of inhibitors in the presence of medium with FBS in triplicate at $37^{\circ} \mathrm{C}$ and put into either normoxic $\left(\mathrm{O}_{2}=21 \%\right)$ or hypoxic $\left(\mathrm{O}_{2}=1 \%\right)$ incubators in parallel for $48 \mathrm{~h}$. Additionally, cells seeded in 96 -well plates $(3,000$ cells/well $)$ were transfected with a small interfering RNA for STAT3 (STAT3 siRNA; Cell Signaling Technology, Beverly, MA, USA) using Lipofectamine 2000 (Invitrogen, Carlsbad, CA, USA) in triplicate, according to the manufacturer's protocol for $48 \mathrm{~h}$, and then cultured under either normoxic or hypoxic conditions for $24 \mathrm{~h}$.

Twenty-five milliliters of Thiazolyl Blue Tetrazolium (Sigma, St. Louis, MO, USA) was added to each sample and plates were further incubated for 4 hours. After that, $100 \mu \mathrm{l}$ of $N, N$-dimethylformamide (Sigma) solubilizing agent was added to each well and plates were placed on a shaker overnight at room temperature. On the following day, the absorbance was read at $595 \mathrm{~nm}$. $\mathrm{IC}_{50}$ against cancer cell lines were calculated for LLL12 and LY5 using GraphPad Prism 7 (GraphPad Software, Inc.).

Colony formation. Cells were grown in six-well cell culture plates and treated with different concentrations of LLL12 and LY5 for the determined timepoint under both normoxic and hypoxic conditions. After trypsinization, the viable cells were collected and seeded at 1,000 cells in $6-\mathrm{cm}$ plates and allowed to grow until DMSO-treated control cells reached confluence under normal oxygen conditions. After 2-3 weeks, cells were washed with PBS twice and fixed with cold methanol for 30 min followed by staining with $1 \%$ crystal violet dye (25\% methanol) at room temperature for $1 \mathrm{~h}$. The plates were then rinsed with distilled water and dried prior to scanning to record the colony numbers.

Western blot analysis. HPAC cells were treated in parallel under $21 \% \mathrm{O}_{2}$ and $1 \% \mathrm{O}_{2}$ with LLL12 $(1,2$ and $3 \mu \mathrm{M})$ or DMSO for about 12 hours. After these treatments, cells were washed with cold PBS, lysed on ice in a modified RIPA buffer [1\% Triton X-100, $1 \%$ deoxycholate, $0.1 \%$ sodium dodecyl sulfate (SDS)] containing protease inhibitors ( $1 \mathrm{mM}$ phenylmethanesulfonyl fluoride) and subjected to SDS-polyacrylamide gel electrophoresis (PAGE). Proteins were transferred onto polyvinylidene difluoride membranes and probed with a 1:1,000 dilution of antibodies against phosphospecific STAT3 (tyrosine 705), phospho-independent STAT3, phospho-specific protein kinase B (P-AKT, threonine 308) and phospho-specific ERK1/2 (threonine 202/tyrosine 204), BCL-XL, survivin, MYC, and glyceraldehyde-3-phosphate dehydrogenase (GAPDH). After incubation with the primary antibodies, HRPconjugated secondary antibodies were added onto the samples with minimal shaking for $90 \mathrm{~min}$. The specific proteins were detected using enhanced chemiluminescence.

Statistical analysis. Student $t$-test was used for statistical analysis and $p$-value determination via Prism 7.0 (GraphPad Software, Inc, La Jolla, CA, USA). Differences were considered statistically significant at $p<0.05$.

\section{Results}

The ability of STAT3 inhibitors to inhibit cell viability was reduced under hypoxic conditions. In order to examine the effects of hypoxia on the activity of STAT3 inhibitors, human cancer cell lines were treated with increasing concentrations of inhibitors for $48 \mathrm{~h}$ under either normoxic or hypoxic conditions and the $\mathrm{IC}_{50}$ concentrations were determined. The results of LLL12 and LY5 are presented in Table I. The $\mathrm{IC}_{50}$ values against human pancreatic cancer, medulloblastoma, and sarcoma cell lines treated in $1 \% \mathrm{O}_{2}$ were all higher than those treated in $21 \% \mathrm{O}_{2}$, increasing by about 2-5-fold. Astonishingly, the $\mathrm{IC}_{50}$ for UW288-1 cells was increased 16-fold under hypoxia compared with 
A

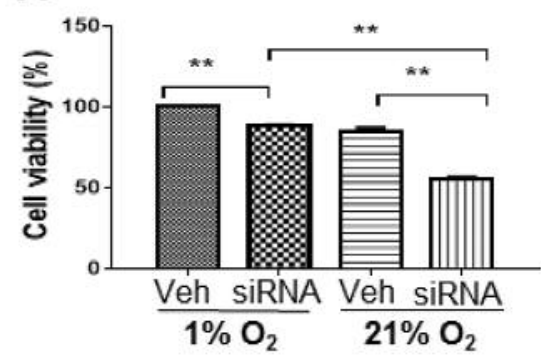

B

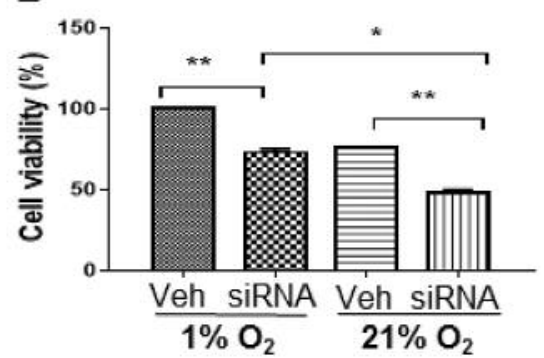

C

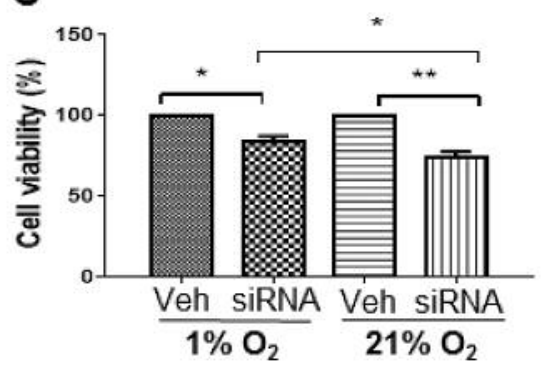

Figure 1. The effect of signal transducer and activator of transcription 3 (STAT3) knockdown by siRNA on inhibition of cancer cell viability under hypoxic compared with normoxic conditions. PANC-1 (A), SaoS2 (B), and HPAC (C) cells were transfected using Lipofectamine 2000 with or without STAT3 siRNA for $48 \mathrm{~h}$ then cultured under normoxic or hypoxic conditions for $24 \mathrm{~h}$, respectively. Methylthiazolyldiphenyl-tetrazolium bromide assays were performed to analyze cell viability. Hypoxia reduced the effect of STAT3 siRNA allowing greater cell survival. Data are the mean \pm SD. The results were representative of three independent experiments. Student $t$-test was used for statistical analysis and p-value determination using Prism 7. Differences were considered statistically significant at ${ }^{*} p<0.05$ and $* * p<0.01$. Veh: Vehicle, siRNA: STAT3 siRNA.

normoxia. Thus, these results suggest that sensitivity to STAT3 inhibitors (LLL12 and LY5) in human cancer cells is reduced under hypoxia.

As seen in Figure 1, STAT3 siRNA transfection of cells reduced viability among under the same oxygen concentrations, indicating the effectiveness of siRNA. However, STAT3 siRNA more significantly reduced cell viability under normoxic conditons than hypoxic conditions. These results indicate that hypoxic conditions might allow greater cell survival under treatment with STAT3 inhibitors or STAT3 siRNA.

Hypoxic conditions reduced STAT3 inhibition of colony formation by human cancer cells. We examined the ability of cancer cells to survive and form colonies after STAT3 inhibitor treatment under hypoxic conditions. Capan-1, HPAF-II, HPAC pancreatic cancer cell lines and UW288, UW426 medulloblastoma cell lines were treated with LLL12 at serial concentrations under $1 \% \mathrm{O}_{2}$ or $21 \% \mathrm{O}_{2}$. The results showed colony numbers were significantly less reduced under hypoxic than under normoxic conditions, especially at low drug concentration. As shown in Figure 2, all human cancer cell lines under normal oxygen concentrations $(21 \%$ $\mathrm{O}_{2}$ ) showed a dramatic decrease of colony numbers, implying reduced ability to recover and form colonies with increasing concentration of LLL12. However, under hypoxic conditions, multiple pancreatic cancer and medulloblastoma cell lines did not show a marked decrease in colony numbers. The difference of colony-forming ability under normoxic and hypoxic conditions was more dramatic when the drug concentration was 0.5 or $1.0 \mu \mathrm{M}$. This was particularly obvious in Capan-1, HPAF-II pancreatic cancer cells and UW426 medulloblasoma cells, in which LLL12treated cells showed little or no colony formation under normoxic conditions, but colony reduction was not significant under hypoxic conditions.

The effects of LY5 on colony formation by SJSA, UW288-1 and UW426 cells were further tested. LY5 significantly inhibited colony-forming ability in all cancer cell lines even at $1.0 \mu \mathrm{M}$ under $21 \% \mathrm{O}_{2}$ (Figure 3). When 2.0 and $3.0 \mu \mathrm{M}$ of LY5 were used under $21 \% \mathrm{O}_{2}$, no visible colonies were observed. In contrast, the ability of LY5 to inhibit colony-forming activity was poor in SJSA sarcoma cells even when 2.0 and $3.0 \mu \mathrm{M}$ were used under hypoxic conditions. Consistently, the ability of LY5 to inhibit colony formation was also reduced in UW288-1 and UW426 medulloblatoma cells under hypoxia. The results from these two different STAT3 inhibitors indicate that hypoxic conditions appear to generate resistance to STAT3 inhibitors in human cancer cells.

Expression of P-STAT3 was increased under hypoxic conditions. The molecular mechanisms through which hypoxia in tumor cells contributes to resistance of STAT3 inhibitors remain largely unknown. HPAC pancreatic cells were subjected to western blot analysis after LLL12 treatment at different concentrations in either $21 \% \mathrm{O}_{2}$ or $1 \%$ $\mathrm{O}_{2}$. As reported, a high level of phosphorylated STAT3 protein (P-STAT3), i.e activated STAT3, contributes to cell survival and proliferation (7). From the results shown in Figure 4 , it can be clearly seen that P-STAT3 levels in cells were higher with LLL12 in $1 \% \mathrm{O}_{2}$ compared with those cultured in $21 \% \mathrm{O}_{2}$. The expression of total STAT3 was similar and slightly decreased as LLL12 concentration increased, indicating that hypoxia may contribute to the inhibitory ability of STAT3 inhibitors. Increased P-STAT3 under hypoxic conditions indicates that higher concentrations 


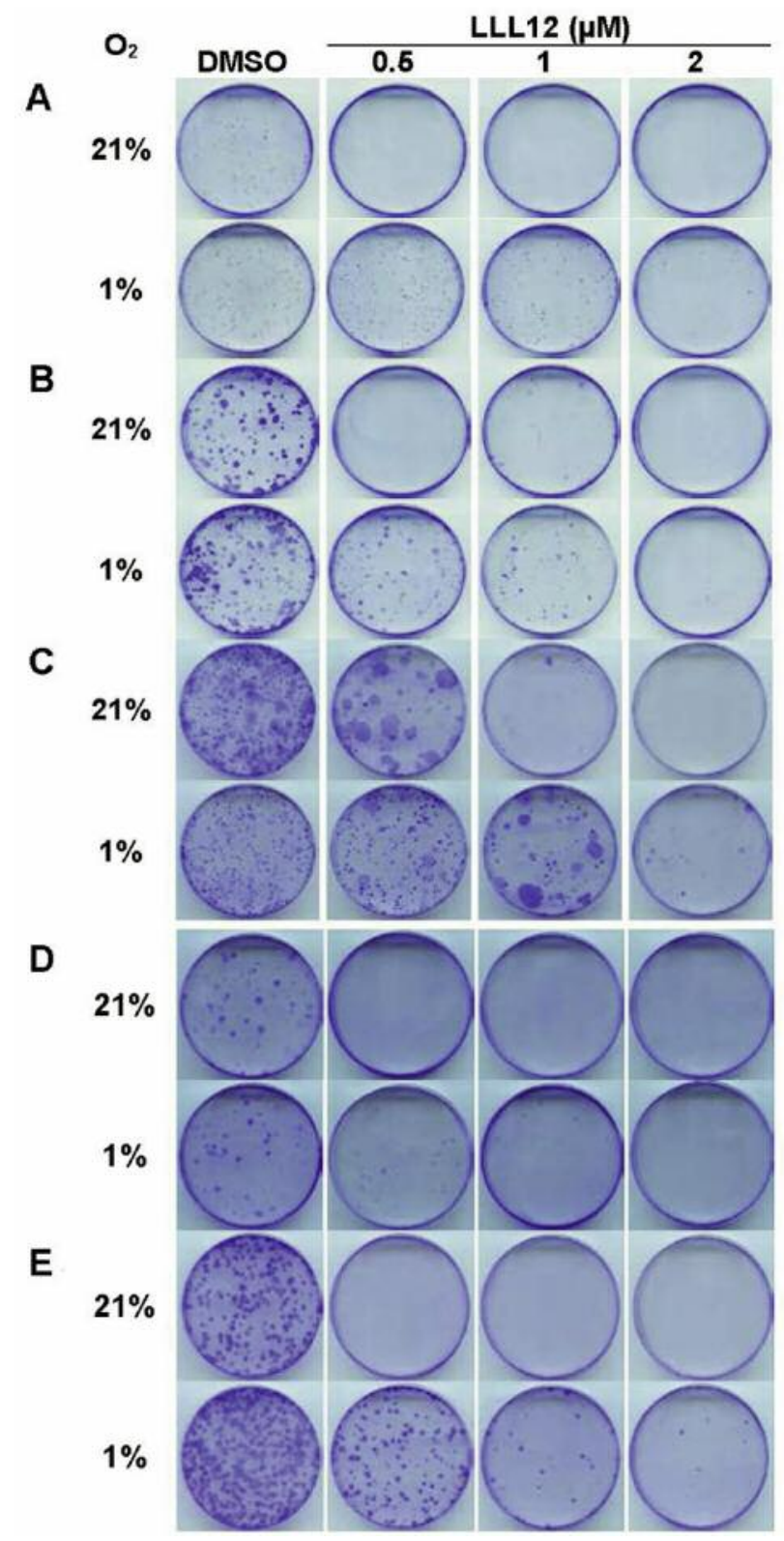

Figure 2. Efficacy of the signal transducer and activator of transcription 3 (STAT3) inhibitor LLL12 to inhibit colony-forming ability of pancreatic cancer cells and medulloblastoma cells under hypoxic compared to normoxic conditions. Human pancreatic cancer cells Capan-1 (A), HPAFII (B), HPAC (C) and medulloblastoma cells UW288-1 (D) and UW426 (E) were treated with $L L L 12$ in $21 \% \mathrm{O}_{2}$ (normoxia) or $1 \% \mathrm{O}_{2}$ (hypoxia) respectively, at the indicated concentrations. After treatment, viable cells were counted and the same cell numbers were seeded then cultured under normoxic conditions for about 2-3 weeks. Colonies were fixed by ice-cold methanol and were stained by crystal violet solution. Hypoxia reduced inhibition of colony formation by STAT3.

of STAT3 inhibitors may be required to be effective and thus hypoxia could be associated with resistance to STAT3 inhibitors. In addition, we did not observe P-AKT and P-

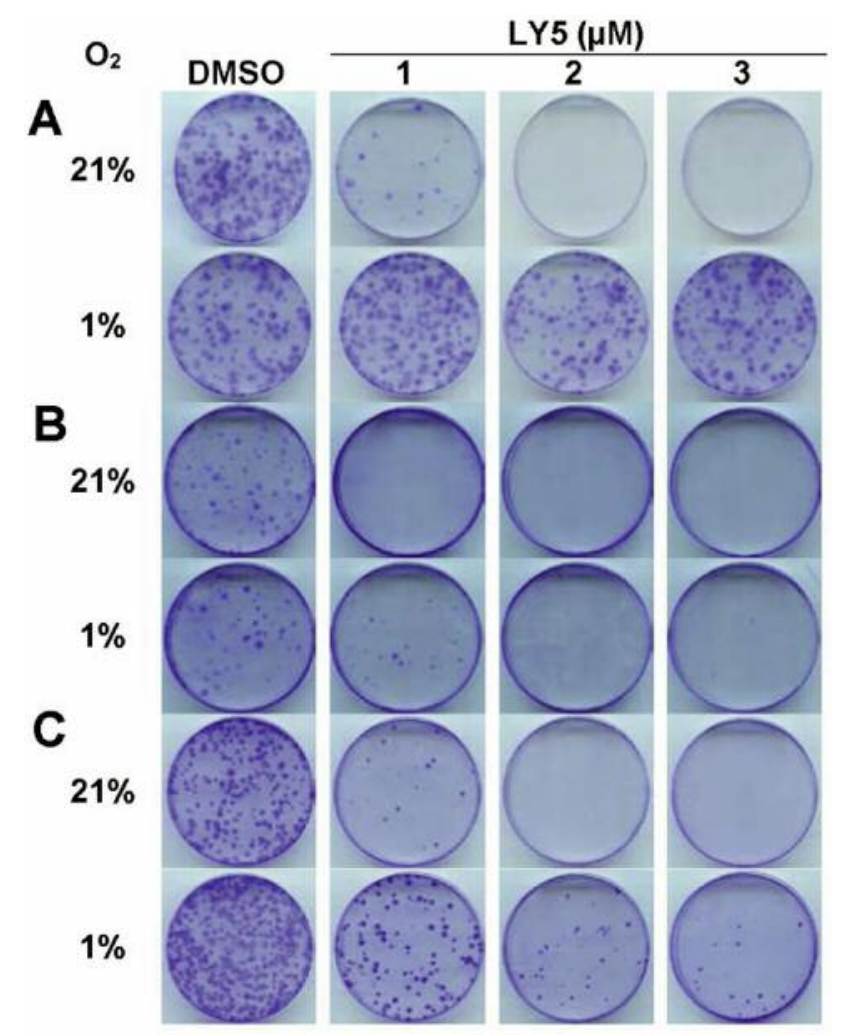

Figure 3. The efficacy of the signal transducer and activator of transcription 3 (STAT3) inhibitor LY5 in inhibiting colony formation by cancer cells under hypoxic with normoxic conditions. Human cancer cell lines SJSA (A), UW288-1 (B) and UW426 (C) were treated with LY5 in $21 \% \mathrm{O}_{2}$ (normoxia) or $1 \% \mathrm{O}_{2}$ (hypoxia), respectively, at the indicated concentrations. STAT3 efficacy was reduced by hypoxia.

ERK increase under hypoxic conditions and in fact, their phosphorylation was reduced (Figure 4). These results suggest AKT and ERK pathways are not involved in resistance to STAT3 inhibitors under hypoxic conditions.

P-STAT3 binds promoter regions in order to regulate the transcription of downstream target genes, which could contribute to uncontrolled proliferation of cancer cells through promoting cell-cycle progression, inhibiting apoptosis, stimulating tumor angiogenesis and metastasis. Accordingly, we examined the expression of STAT3 downstream targets cMYC, survivin and BCL-XL. As shown in Figure 4, BCL-XL and survivin protein levels were slightly down-regulated when cells were treated with LLL12, both under hypoxic and under normoxic conditions, which suggests that these two downstream target genes may not be involved in resistance to LLL12 under hypoxic conditions. Interestingly, c-MYC expression was diminished in cells treated with 2.0 and 3.0 $\mu \mathrm{M}$ of LLL12 under normoxic conditions, while expression was sustained when cells were treated with 2.0 and $3.0 \mu \mathrm{M}$ of LLL12 under hypoxic conditions. 


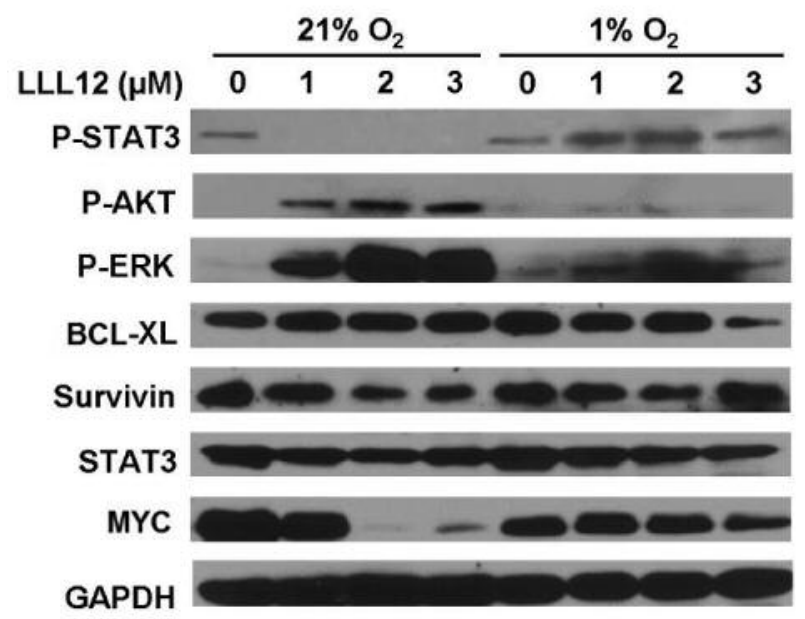

Figure 4. Western blot analysis of phospho-specific signal transducer and activator of transcription 3 (P-STAT3), STAT3, phospho-specific protein kinase $B(P-A K T)$, phospho-specific extracellular-regulated protein kinase ( $P$-ERK), B-cell lymphoma $(B C L)$-XL, survivin, myelocytomatosis (MYC) and glyceraldehyde-3-phosphate dehydrogenase (GAPDH) in HPAC cells treated with LLL12 at concentrations of 1, 2, $3 \mu M$ under normoxic $(21 \%$ $\left.\mathrm{O}_{2}\right)$ and hypoxic $\left(1 \% \mathrm{O}_{2}\right)$ conditions. STAT3 activation increased as reflected in increasing P-STAT3 under hypoxic conditions, although total STAT did not appear to differ significantly.

\section{Discussion}

STAT3 is a transcription factor with important roles in oncogenesis and cancer progression, associated with cancer cell proliferation, inhibition of apoptosis, immune escape, epithelial-mesenchymal transition, invasion, and angiogenesis $(6,7)$. Tumor hypoxia represents an independent prognostic factor and always acts as an important mediator of resistance to cancer treatment. Cells undergo a variety of biological responses and adaptive changes that allow them to survive and even proliferate when placed in hypoxic conditions, which contribute to tumor progression, aggressiveness and chemoresistance (20). For example, it has been reported that endostatin, one of the most widely used anti-angiogenic drugs, was unable to reduce tumor volume, which may well be due to increased transforming growth factor $\beta 1$ levels and increased hypoxia (21). The network between hypoxia and drug resistance is complicated. The mechanisms of the effects of hypoxia on cancer cells may involve the induced expression of various growth factors which are known to promote cell proliferation, and the up-regulation of genes involved in drug resistance including multiple drug resistance genes encoding the export pump P-glycoprotein in hypoxic cells (22).

The effects of STAT3 inhibitors on cancer cells under hypoxic conditions is poorly understood. Our study has shown that the STAT3 inhibitors LLL12 and LY5 exhibited potent activity under normoxic conditions. However, hypoxic conditions reduced the ability of STAT3 inhibitors and of STAT3 siRNA to inhibit cell viability. The increase of $\mathrm{IC}_{50}$ indicates increased cancer cell survival and enhanced resistance to STAT3 inhibitors under hypoxic conditions. Hypoxic conditions reduced the ability of STAT3 inhibitors to inhibit colony formation of human cancer cells here, suggesting that cancer cell survival and proliferation might still be partially maintained under hypoxic conditions even in the presence of STAT3 inhibitors. Our results from western blot analysis additionally demonstrated that P-STAT3 levels were maintained and even increased under hypoxic conditions relative to those under normoxic conditions when cancer cells were treated with a STAT3 inhibitor. Activated STAT3 may contribute to resistance to cancer drugs (23). In addition, there is a feedback activation of STAT3 as a possible cancer drug-resistance mechanism (24). Inhibition of STAT3 caused increased expression of P-AKT and PERK under normoxic, but not under hypoxic conditions. The mechanism for this is not clear, but might be due to feedback activation of RTKs, which result in elevated PAKT and P-ERK under normoxic conditions. The expression of P-AKT and P-ERK was reduced under hypoxia, suggesting that resistance to STAT3 inhibitors is more likely due to STAT3 signaling rather than AKT and ERK. There was no obvious increase in BCL-XL and survivin, suggesting that these two downstream effectors of STAT3 may not play a role in resistance to STAT3 inhibitors. Interestingly, c-MYC expression was mostly maintained in the presence of STAT3 inhibitor under hypoxic conditions, suggesting that c-MYC might contribute to cancer cell survival/proliferation when cells are treated with inhibitor under hypoxic conditions.

It has been reported that hypoxia may activate STAT3 via various mechanisms, including the activation of sarcoma kinase, activation of Janus kinase 2, activation of nuclear factor-kB and activator protein-1 (25), suggesting that STAT3 can be acted on by HIF $1 \alpha$ either dependently or independently (26). For example, STAT3 was found to be a potential modulator of HIF1-mediated vascular endothelial growth factor expression (27), and it could then regulate cell survival, proliferation, angiogenesis and cell migration (28). Thus, the activation of STAT3 proteins under hypoxic conditions may play an important role in tumor drug resistance. However, further studies are still needed to elucidate additional mechanisms of cancer cell resistance to STAT3 inhibitors under hypoxic conditions in order to develop better therapeutic strategies to target STAT3 signaling.

\section{Competing Interests}

The Authors declare that they have no competing interests. 


\section{Acknowledgements}

This research was supported by NIH/NINDS 1R01NS087213-01A1 grant and the University of Maryland School of Medicine and Comprehensive Cancer Center start-up fund.

\section{References}

1 Ihle JN: STATs: Signal transducers and activators of transcription. Cell 84(3): 331-334, 1996.

2 Mui AL: The role of STATs in proliferation, differentiation, and apoptosis. Cell Mol Life Sci 55(12): 1547-1558, 1999.

3 Bromberg JF, Wrzeszczynska MH, Devgan G, Zhao Y, Pestell RG, Albanese C and Darnell JE Jr.: STAT3 as an oncogene. Cell 98(3): 295-303, 1999.

4 Bowman T, Garcia R, Turkson $\mathrm{J}$ and Jove R: STATs in oncogenesis. Oncogene 19(21): 2474-2488, 2000.

5 Klampfer L: The role of signal transducers and activators of transcription in colon cancer. Front Biosci 13: 2888-2899, 2008.

6 Germain D and Frank DA: Targeting the cytoplasmic and nuclear functions of signal transducers and activators of transcription 3 for cancer therapy. Clin Cancer Res 13(19): 5665-5669, 2007.

7 Yu H, Lee H, Herrmann A, Buettner R and Jove R: Revisiting STAT3 signalling in cancer: new and unexpected biological functions. Nat Rev Cancer 14(11): 736-746, 2014.

8 Kusaba T, Nakayama T, Yamazumi K, Yakata Y, Yoshizaki A, Inoue K, Nagayasu T and Sekine I: Activation of STAT3 is a marker of poor prognosis in human colorectal cancer. Oncol Rep 15(6): 1445-1451, 2006.

9 Yakata Y, Nakayama T, Yoshizaki A, Kusaba T, Inoue K and Sekine I: Expression of p-STAT3 in human gastric carcinoma: significant correlation in tumour invasion and prognosis. Int $\mathrm{J}$ Oncol 30(2): 437-442, 2007.

10 Miklossy G, Hilliard TS and Turkson J: Therapeutic modulators of STAT signalling for human diseases. Nat Rev Drug Discov 12(8): 611-629, 2013.

11 Lin L, Hutzen B, Li PK, Ball S, Zuo M, DeAngelis S, Foust E, Sobo M, Friedman L, Bhasin D, Cen L, Li C and Lin J: A novel small molecule, LLL12, inhibits STAT3 phosphorylation and activities and exhibits potent growth-suppressive activity in human cancer cells, Neoplasia 12(1): 39-50, 2010.

12 Liu A, Liu Y, Li PK, Li C and Lin J: LLL12 inhibits endogenous and exogenous interleukin-6-induced STAT3 phosphorylation in human pancreatic cancer cells. Anticancer Res 31(6): 2029-2036, 2011.

$13 \mathrm{Yu}$ W, Xiao H, Lin J and Li C: Discovery of novel STAT3 small molecule inhibitors via in silico site-directed fragment-based drug design. J Med Chem 56(11): 4402-4412, 2013.

14 Xiao H, Bid HK, Jou D, Wu X, Yu W, Li C, Houghton PJ and Lin J: A novel small molecular STAT3 inhibitor, LY5, inhibits cell viability, cell migration, and angiogenesis in medulloblastoma cells. J Bio Chem 290(6): 3418-3429, 2015.

15 Koong AC, Mehta VK, Le QT, Fisher GA, Terris DJ, Brown JM, Bastidas AJ and Vierra M: Pancreatic tumors show high levels of hypoxia. Int J Radiat Oncol Biol Phys 48(4): 919-922, 2000.
16 Lin A and Maity A: Molecular patgways: a novel approach to targeting hypoxia and improving radiotherapy efficacy via reduction in oxygen demand. Clin Cancer Res 21(9): 1995-2000, 2015.

17 Wilson WR and Hay MP: Targeting hypoxia in cancer therapy. Nat Rev Cancer 11(6): 393-410, 2011.

18 Syu JP, Chi JT and Kung HN: NRF2 is the key to chemotherapy resistance in MCF7 breast cancer cells under hypoxia. Oncotarget 7(12): 14659-14672, 2016.

19 He X, Wang J, Wei W, Shi M, Xin B, Zhang T and Shen X: Hypoxia regulates ABCG2 activity through the activivation of ERK1/2-HIF1 $\alpha$ and contributes to chemoresistance in pancreatic cancer cells. Cancer Biol Ther 17(2): 188-198, 2016.

20 Ruan K, Song G and Ouyang G: Role of hypoxia in the hallmarks of human cancer. J Cell Biochem 107(6): 1053-1062, 2009.

21 Wang Y, Jiang M, Li Z, Wang J, Du C, Yanyang L, Yu Y, Wang X, Zhang N, Zhao M, Wang L, Li M and Luo F: Hypoxia and TGF- $\beta 1$ lead to endostatin resistance by cooperatively increasing cancer stem cells in A549 transplantation tumors. Cell Biosci 5: $72,2015$.

22 Jeong JK, Gurunathan S, Kang MH, Han JW, Das J, Choi YJ, Kwon DN, Cho SG, Park C, Seo HG, Song H and Kim JH: Hypoxia-mediated autophagic flux inhibits silver nanoparticletriggered apoptosis in human lung cancer cells. Sci Rep 6: 21688, 2016

23 Tan FH, Putoczki TL, Stylli SS and Luwor RB: The role of STAT3 signaling in mediating tumor resistance to cancer therapy. Curr Drug Targets 15(14): 1341-1353, 2014.

24 Zhao C, Li H, Lin HJ, Yang S, Lin J and Liang G: Feedback activation of STAT3 as a cancer drug-resistance mechanism. Trends Pharmacol Sci 37(1): 47-61, 2016.

25 Pawlus MR, Wang L and Hu CJ: STAT3 and HIF1 $\alpha$ cooperatively activate HIF1 target genes in MDA-MB-231 and RCC4 cells. Oncogene 33(13): 1670-1679, 2014.

26 Doktorova H, Hrabeta J, Khalil MA and Eckschlager T: Hypoxia-induced chemoresistance in cancer cells: the role of not only HIF-1. Biomed Pap Med Fac Univ Palacky Olomouc Czech Repub 159(2): 166-177, 2015.

27 Jung JE, Lee HG, Cho IH, Chung DH, Yoon SH, Yang YM, Lee JW, Choi S, Park JW, Ye SK and Chung MH: STAT3 was a potential modulator of HIF-1-mediated VEGF expression in human renal carcinoma cells. FASEB J 19(10): 1296-1298, 2005.

28 Kang SH, Yu MO, Park KJ, Chi SG, Park DH and Chung YG: Activated STAT3 regulates hypoxia-induced angiogenesis and cell migration in human glioblastoma. Neurosurgery 67(5): 1386-1395, 2010.
Received November 2, 2016

Revised December 14, 2016

Accepted December 15, 2016 